\title{
Arbor
}

\section{Un relato: la colección Thyssen-Bornemisza en España}

\author{
Rodrigo Uría Meruéndano
}

Arbor CLXV, 649 (Enero 2000), 91-101 pp.

La historia de la venida a España de la Colección Thyssen-Bornemisza comienza para mí en abril de 1986, coincidiendo con la inauguración en la antigua sala de exposiciones de la Biblioteca Nacional de la muestra de Maestros Modernos de dicha Colección. Con tal motivo, el entonces Ministro de Cultura, Don Javier Solana, me invitó a almorzar al Ministerio con los Barones Thyssen y otras personas. Estaba yo por aquel entonces terminando un encargo que había recibido del Gobierno, también en el ámbito de la cultura, cual fué la recuperación del cuadro de Goya exportado ilegalmente de España, la «Marquesa de Santa Cruz», operación en la que llevaba yo enzarzado varios meses colaborando con el entonces Director General de Bellas Artes y posterior Subsecretario de Cultura, Don Miguel Satrústegui. Unos días antes del citado almuerzo, se había conseguido que un Juez inglés paralizase la subasta de la obra en la casa Christie's de Londres. Tal decisión judicial, aún de carácter cautelar, tuvo el fulminante efecto de sentar a la mesa de la negociación al «propietario aparente» del referido cuadro de Goya, negociación en la que llegamos a la fijación de un «precio indemnizatorio» que debería ser pagado por el Estado Español al referido «propietario aparente» inferior en mucho, naturalmente, al valor de mercado de la obra. Pero como en el Ministerio de Cultura no existía partida presupuestaria alguna que cubriese la compra de «La Marquesa de Santa Cruz», el Ministro Solana tomó la decisión de acudir al patrocinio privado. $\mathrm{Y}$ todo ello salió a colación en el almuerzo con los Barones Thyssen a que me vengo refiriendo. La reacción del Barón Thyssen fué de gran interés en el asunto ya que, según nos explicó, estaba comenzando a organizar una exposición en su Museo de «Villa Favorita» en Lugano de cuadros de Goya de propiedad privada en la 
cual, lógicamente, la presencia de nuestra Marquesa, una obra de gran calidad, curiosa, «desaparecida» durante mucho tiempo y, desde luego, muy "glamorosa», daría a esa exposición de Goyas privados un interés especialísimo. Inmediatamente después, Miguel Satrústegui y yo mismo establecimos conversaciones con los Barones Thyssen para conseguir que contribuyeran económicamente a la recuperación del cuadro. $\mathrm{Al}$ cabo de varias reuniones, se consiguió que el Barón Thyssen aceptara sufragar la mitad del precio indemnizatorio convenido con el «propietario aparente» inglés; pero el Barón Thyssen estableció una condición que el Estado Español no podía aceptar: la co-propiedad del cuadro. Así se lo dijimos a los Barones Thyssen, Miguel Satrústegui y yo mismo en una cena en su casa de Oxfordshire en octubre de 1986, pensando que allí terminaban las conversaciones entre el Gobierno y ellos mismos en relación con la «Marquesa de Santa Cruz». Pero cual no sería nuestra sorpresa cuando Carmen Cervera, Baronesa Thyssen-Bornemisza, nos dijo que aunque ello fuera así, podríamos hablar de algo más importante y, después de explicarnos las grandes dificultades que tenían para albergar toda su colección en el Museo de "Villa Favorita» en Lugano, afirmó que estaban pensando seriamente en España como sede de su colección. Por la ilusión y el énfasis con que se expresó, me dí cuenta, en aquel momento ya, de que España contaba con la más formidable aliada.

Pasaron muchos meses sin que yo volviera a oir hablar del asunto pero sé que durante esos meses Luis Gómez de Acebo, Duque de Badajoz, había puesto al servicio de la operación su rápida inteligencia, su extraordinaria simpatía y sus dotes diplomáticas. Nunca podremos agradecer los españoles bastante al Duque de Badajoz todo lo que hizo durante aquellos meses y los años que les siguieron. La realidad es que sus gestiones y la pasión por España de Carmen Cervera consiguieron convencer al Barón Thyssen y a sus herederos sobre la conveniencia de que la Colección viniese a nuestro país.

Lo cierto es que vuelvo a tomar contacto con el asunto en abril de 1987 cuando en el Ministerio de Cultura me muestran una carta del Duque de Badajoz en nombre del Barón Thyssen dirigida al Gobierno Español en la cual se mencionaba la posibilidad de ceder la propiedad de parte de la Colección al Estado y ceder la posesión del resto en régimen de préstamo de larga duración. Recibí entonces el encargo profesional de asesorar jurídicamente al Ministerio en la operación y de actuar de co-negociador (junto con los representantes de ese Ministerio) en relación con la misma. Inmediatamente, en mayo de 1987, preparamos una contestación a la carta del Duque de 
Un relato: La colección Thyssen Bornemisza en España

Badajoz que podríamos llamar la primera oferta informal del Gobierno Español y en ella se menciona ya la cesión del Palacio de Villahermosa para albergar la Colección. Fué ésta una decisión ciertamente valiente del Ministro Solana, puesto que el Palacio de Villahermosa estaba ya asignado al Museo del Prado a efectos de facilitar la ampliación del mismo. Pero a pesar de ello, el Gobierno pensó que sería imposible encontrar un lugar más adecuado para instalar la Colección Thyssen si es que algún día venía a España y, sobre todo, entendió que la cesión del Palacio de Villahermosa era una baza importantísima a la hora de competir con las otras ofertas que ya se anunciaban y traer la Colección a nuestro país. En segundo lugar, se mencionaba en aquella carta que la entidad que recibiría la Colección sería una Fundación Cultural Privada. ¿Por qué una fundación privada y no el propio Estado? Porque la oferta tenía que ser competitiva y consecuentemente muy imaginativa; porque la oferta de España tenía que ser muy solvente; y porque era evidente que el régimen previsto de cesión de una parte de la Colección y recepción de otra parte en régimen de préstamo entrañaba que entre el Estado Español y los propietarios de la Colección, la familia Thyssen, iba a haber una serie de relaciones permanentes; no se trataba de una compraventa normal en la que una vez recibido el precio, el vendedor pierde toda relación con la cosa vendida. Era algo mucho más complejo, y había que crear un instrumento jurídico en el que las dos partes, la parte compradora y la parte vendedora, la parte prestadora y la parte prestataria, pudieran compartir un «terreno de juego» en el que, a través de los órganos de una institución con presencia de ambos, pudiese haber una comunicación y un diálogo fluido entre ambas a lo largo de toda la operación. Su Majestad El Rey Juan Carlos I no se opuso a que la Fundación, una vez constituída, impetrase su alto patrocinio y fué, desde el primer momento, gustoso campeón de la operación proyectada.

Desde mayo de 1987 hasta abril de 1988, hubo constantes conversaciones y negociaciones entre el Barón Thyssen y los «Trustees», administradores del «Trust» propietario de las obras que le daban vueltas a la oferta española y a otras ofertas competidoras: Stüttgart, Bonn, París -que, al parecer, ofrecía el Petit Palais- y, sobre todo, la Fundación Getty de Malibú (California) que hizo una oferta importantísima por la compra de la Colección. Pero lo cierto es que esta última oferta, por alta que fuera, implicaba una compraventa pura y dura: la familia Thyssen y, sobre todo, el Barón Thyssen-Bornemisza, recibirían el histórico cheque pero tendrían que separarse para siempre de la Colección 
que había hecho su padre y que él había complementado con tan extraordinario acierto.

En cambio, la oferta española suponía algo cardinal para la Colección: su exhibición enfrente del Museo del Prado y muy cerca del Reina Sofía en medio de la llamada «milla de oro» museística de Madrid. No obstante, por la parte Thyssen existían ciertas reticencias y alguna desconfianza sobre la capacidad española para cumplir las promesas que se hacían. Se pensaba que en este país no había suficiente capacidad de gestión cultural para, en el tiempo que se ofrecía, conseguir el dinero necesario, hacer la rehabilitación del Palacio de Villahermosa y proceder con suficiente pericia a la delicada operación del transporte de tantos cuadros. Había el temor de que todo eso se hiciese de una manera más bien «latina» e improvisada. Sin embargo, el modelo español ofreció desde el primer momento a los propietarios de la Colección y muy principalmente al Barón y la Baronesa Thyssen, un modelo de organización común en el cual ellos podrían seguir estando presentes y colaborar con el Gobierno Español en las muchas cosas que había que hacer.

En abril de 1988, superadas las reticencias iniciales, se firmó un primer Protocolo de Intenciones entre el Ministro de Cultura y el Barón Thyssen y se dió así el pistoletazo de salida para la operación de préstamo de la práctica totalidad de la Colección. ¿Por qué ahora préstamo y antes cesión de la propiedad? Pues porque la parte Thyssen, actuando de forma extraordinariamente prudente y, desde luego, muy conservadora, prefirió hacer una operación de préstamo a largo plazo (algo menos de diez años) para así no comprometerse definitivamente y poder comprobar si los españoles éramos capaces de cumplir lo que prometíamos. Persistía, pues, alguna desconfianza. Aquel momento, el de la firma de la Carta de Intenciones definiendo la operación como de préstamo, fué un momento ciertamente difícil ya que en el mundo de la cultura, en algunos medios de comunicación e incluso en ciertas instancias gubernamentales, se pensaba en la adquisición definitiva de la propiedad de la Colección. Pero las instancias políticas decisorias no se arredraron y seguimos negociando durante varios meses un texto de contrato de préstamo a nueve años y seis meses. Fué un documento contractual complejísimo, sometido al Derecho inglés y redactado en consecuencia -con su habitual prolijidad- por abogados ingleses. Las negociaciones sobre ese texto inicial fueron muy duras e intensas. Por fin, en diciembre de 1988, llegamos a un texto común, se firmó el Contrato de Préstamo por el tiempo referido con una contraprestación económica por España de cinco millones de dólares anuales y se cons- 
Un relato: La colección Thyssen Bornemisza en España

tituyó la Fundación Cultural Privada («Fundación Colección ThyssenBornemisza») a la que el Gobierno Español aportó nueve mil millones de pesetas y el Barón Thyssen-Bornemisza una peseta simbólica. De los nueve mil millones aportados por el Estado, siete mil millones servirían para atender el pago de los cánones del préstamo durante los nueve años y seis meses y dos mil millones para las obras de rehabilitación del Palacio de Villahermosa. Posteriormente, se vió, como suele suceder con las obras, cómo la rehabilitación del Palacio sobrepasaba la previsión inicial. Aquella Fundación tenía un Patronato paritario, donde estábamos cinco personas que representábamos al Gobierno y otras cinco que representaban a los propietarios de la Colección. En sus Estatutos, se requería la unanimidad para todas las cosas realmente importantes en la vida de la Fundación como, por ejemplo, la aprobación de los presupuestos anuales, el control de las obras de rehabilitación del Palacio de Villahermosa, la modificación de los Estatutos, el nombramiento de Comités Ejecutivos y el nombramiento de Conservador Jefe, siendo el de Director Gerente privativo del Gobierno.

Aunque sólo sea a título anecdótico, hay que decir que, firmado el Protocolo de Intenciones y antes del otorgamiento del Contrato de Préstamo, el Gobierno de Su Majestad Británica se interfirió extemporánea y arbitrariamente en el proceso, con una oferta realmente importante. Ofrecía a los propietarios de la Colección la «National Portrait Gallery» situada en Trafalgar Square, para los Maestros Antiguos, espacio en el Canary Wharf para los Modernos, y una sustanciosa cantidad en libras esterlinas. Parecía como si alguna filtración hubiera proporcionado al Gobierno de la Señora Thatcher información sobre la oferta española ... Las presiones británicas fueron realmente intensas y se produjeron en los niveles más altos. Pero el Barón Thyssen siguió una vez más la imbatible inclinación española de su esposa y, cómo no, hizo honor a las intenciones expresadas en el Protocolo. Esta vez le tocó a Inglaterra perder frente a España. Tan es así, que en sus Memorias la Dama de Hierro inglesa se lamenta, como uno de los puntos negros de su mandato, de que se le «escapara» la Colección Thyssen-Bornemisza.

Durante los años 1989, 1990 y 1991, suceden muchas cosas. La primera, la selección de un arquitecto que redactase el Proyecto de rehabilitación del Palacio de Villahermosa. La elección de la Fundación recayó, como es sabido, sobre Rafael Moneo. Los Barones Thyssen tenían otra idea sobre el arquitecto y pensaban en algunos arquitectos más «vistosos». Afortunadamente, después de una visita al Museo de 
Arte Romano de Mérida, obra emblemática de Rafael Moneo, y teniendo en cuenta que entonces Moneo era Decano de la Facultad de Arquitectura de la Universidad de Harvard, el nombre de Moneo fué aceptado y realizó el proyecto arquitectónico que está a la vista de todos.

Se seleccionaron después, en concurso restringido, contratistas para la realización de las obras y la primera piedra de las mismas se puso el día 13 de marzo de 1990. Las obras se terminan y se presentan al público en mayo de 1992, es decir, un poco más de dos años para llevar a cabo una obra que supuso el vaciamiento completo del edificio, la conservación de sus fachadas y el diseño y construcción del nuevo espacio museístico de acuerdo con la maquetación realizada por los expertos.

Durante los meses de junio y julio de 1992, tuvo lugar la operación de traslado o transporte de pinturas más importante de la historia si se exceptúa el desalojo y realojamiento del Museo del Prado con motivo de la Guerra Civil española. Se transportaron más de novecientas obras de arte en dos meses, cuadros, dibujos, esculturas y objetos artísticos y para cada uno de ellos fué necesario hacer un informe sobre el estado en que se encontraba en el punto de salida y un informe sobre la condición de cada obra de arte en el punto de recepción. La inversión en las cajas que contenían los cuadros más importantes fué de varios cientos millones de pesetas. Cajas, por supuesto, especialmente climatizadas y acondicionadas. Aproximadamente el sesenta y cinco por ciento de las obras se transportaron en aviones especialmente fletados al efecto. El resto, en camiones especialmente acondicionados y custodiados.

En abril de 1992, se firmó un contrato adicional entre el Gobierno y los propietarios de la Colección para que setenta y ocho obras de la misma se mostrasen al público en el Monasterio de Pedralbes de Barcelona, cumpliendo así un deseo muchas veces expresado por Carmen Cervera, Baronesa Thyssen-Bornemisza.

Durante los meses de julio a septiembre de 1992, se colgó la Colección en el Museo sito en el Palacio de Villahermosa. No sólo la altísima calidad del Proyecto de Rafael Moneo sino también la extraordinaria pericia del Conservador-Jefe de la Colección, Tomás Llorens, hicieron que fuese posible el Museo que inauguraron Sus Majestades Los Reyes y el Presidente del Gobierno el día 8 de octubre de 1992.

España había cumplido. En tiempo, en dinero, en calidad, en gusto, en competencia científica y técnica, en todo. Fuí testigo de cómo, en aquel memorable 8 de octubre, muchas personas no creían lo que veían. 
Un relato: La colección Thyssen Bornemisza en España

De la Colección, hoy conocida y apreciada universalmente, hay que destacar su extraordinaria complementariedad con las colecciones del Museo del Prado y el resto de las colecciones públicas españolas. En ella están dignamente representados movimientos y momentos de la historia de la pintura muy escasamente presentes en las colecciones españolas: los Maestros Antiguos alemanes, los impresionistas franceses, los expresionistas alemanes, las vanguardias rusas, ...

Piensa este relator que la diligencia y competencia de las que hizo gala España borraron cualquier sombra de reticencia o temor en los propietarios de la Colección. Tan es así, que en marzo de 1992, con el Museo prácticamente terminado aunque no inaugurado, se firmó entre el Ministro de Cultura y los representantes de los propietarios, una nueva Carta de Intenciones relativa, esta vez sí, a la compra de la Colección. Esa carta, firmada en un sólo ejemplar que se depositó en un Notario madrileño fué un secreto bien guardado. En ella se fijaba la cifra que habría que pagar por la Colección para el supuesto de que se llegase al acuerdo de cesión de la propiedad entre los propietarios y el Gobierno de España. La cifra en cuestión era de trescientos cincuenta millones de dólares. En aquel momento, la Colección podría tener un valor de entre mil y mil quinientos millones de dólares, cifra ésta aproximada a la que se llegaba tomando en cuenta los valores de adquisición y aplicando a éstos índices complejos, comparándolos con los valores de seguro, analizando las transacciones en régimen de mercado abierto que se habían producido para obras de similar categoría, etc. Se compraba, pues, en trescientos cincuenta lo que pudiera bien valer mil quinientos y ahí había un indudable elemento de liberalidad por parte de los propietarios que tuvo su contrapartida en la pretensión de los mismos de no separarse completamente de la Colección y de seguir teniendo algo que decir para que se mantuviesen los principios que la habían iluminado desde que la inició el padre del actual Barón Thyssen-Bornemisza, nada más terminar la Primera Guerra Mundial, hasta nuestros días: su unidad, su calidad y su internacionalidad.

Desde la inauguración del Museo en octubre de 1992 los propietarios y el Estado español no cesaron de estar en contacto con el fin de configurar la operación por la que definitivamente la Colección pasaba a ser propiedad de la Fundación española. Los abogados ingleses, suizos, de las Islas Bermudas y españoles de ambas partes trabajamos duro preparando los perfiles de la transacción definitiva y tuvimos listo un contrato de adquisición de la propiedad por la Fundación española al cabo de seis o siete meses. El texto contractual gozó de la aprobación 
de ambas partes y se firmó en junio de 1993, disueltas las Cámaras y previa aprobación por el Consejo de Ministros de un Real Decreto Ley que daba cobertura normativa a tan importante operación y que fué posteriormente ratificado por la Diputación Permanente de las Cortes Generales. El consenso entre el Partido Socialista (en el Gobierno) y el Partido Popular (en la oposición) fué tanto más admirable cuanto que se produjo en plena campaña electoral. El apoyo del Partido Popular a la operación fué absoluto.

Por virtud del contrato de compraventa, la Fundación adquirió la propiedad de setecientos setenta y cinco cuadros que, junto con los integrantes de los préstamos adicionales que hicieron en el momento de la operación de compra el Barón Thyssen y sus herederos, integran la totalidad de los que alberga el Museo. Con motivo de la adquisición de la propiedad de la Colección, se modificaron muy sensiblemente los Estatutos de la Fundación propietaria. Antes, había una paridad de miembros en el Patronato de la misma y desde 1993, hay un Patronato de doce miembros, ocho de los cuáles hemos sido nombrados por el Gobierno y cuatro por la parte antiguamente propietaria. En lo único en lo que hay que contar con el voto favorable de los representantes de los antiguos propietarios es en aquellos aspectos relativos a los «standards» museísticos del Palacio de Villahermosa, una eventual desmembración de la Colección, y ciertas disposiciones tendentes a garantizar la presencia internacional de la misma en todo caso. Preside el Patronato el Ministro de Educación y Cultura y es Vicepresidenta vitalicia, Carmen Cervera, Baronesa Thyssen-Bornemisza. El Estado ha cedido a la Fundación Thyssen-Bornemisza la propiedad del Palacio de Villahermosa.

La actividad y la gestión de la Fundación responde a tres principios: el principio del interés público; el principio de la exquisita conservación y permanente exposición y estudio de las obras de arte; y el principio de la mejor administración empresarial. Y hay que decir que, en el respeto a esos tres principios, la Fundación no lo está haciendo nada mal. Baste un botón de muestra: los ingresos de explotación cubren los gastos en un ochenta por ciento. Pocos, muy pocos museos en el mundo pueden decir lo mismo.

Pero el relato continúa ... Carmen Cervera, Baronesa ThyssenBornemisza, ha venido reuniendo una excelente colección de pintura a base de obras procedentes algunas de donaciones de su esposo $\mathrm{y}$ otras muchas adquiridas en los mercados internacionales y españoles. Algunas de las piezas que la integran ya han sido expuestas al público y muy probablemente cuando este relato se 
publique, habrá otras expuestas en la sala de exposiciones temporales del Museo Thyssen de Madrid.

Siendo deseo de la Baronesa Thyssen-Bornemisza que su propia colección, sin perder su independencia, figure asociada de alguna forma a la colección de su marido, hoy propiedad de la Fundación, la ofreció al Gobierno para que fuese albergada y expuesta, en la medida de lo posible, en el actual Museo. Dado que los espacios disponibles eran claramente insuficientes para amparar dicho proyecto, el Gobierno tomó la decisión de adquirir los dos inmuebles colindantes con el Palacio de Villahermosa para albergar en ese «nuevo Museo» la colección de la Baronesa, exponer las obras más relevantes de la misma, creando un todo armónico entre los tres edificios que permita que las dos colecciones puedan ser expuestas contando con las superficies necesarias para espacios públicos suficientes de exposiciones temporales, estudio y recreo. El préstamo de la colección de la Baronesa tendrá una duración de once años desde la firma del oportuno contrato y no acarrea el pago de cantidad dineraria alguna por la Fundación prestataria o por el Gobierno. La rehabilitación de los nuevos edificios no durará menos de dos años contados a partir de la toma de posesión de los mismos por parte de la Fundación.

No parece aventurado afirmar que la gran operación cultural relatada, en la que han estado implicados cuatro Ministros de Cultura socialistas y dos Ministros de Cultura pertenecientes al Partido Popular se ha convertido en un ejemplo admirable y admirado internacionalmente de cómo incrementar el patrimonio histórico-artístico de una nación y, desde luego, en un modelo de gestión cultural que, respetando siempre el interés público, aplica criterios de actuación dinámicos e innovadores. 


\section{Rodrigo Uría Meruéndano}

FIGURA 1. «Fränzi ante una silla tallada» Ernst Ludwig Kirchner

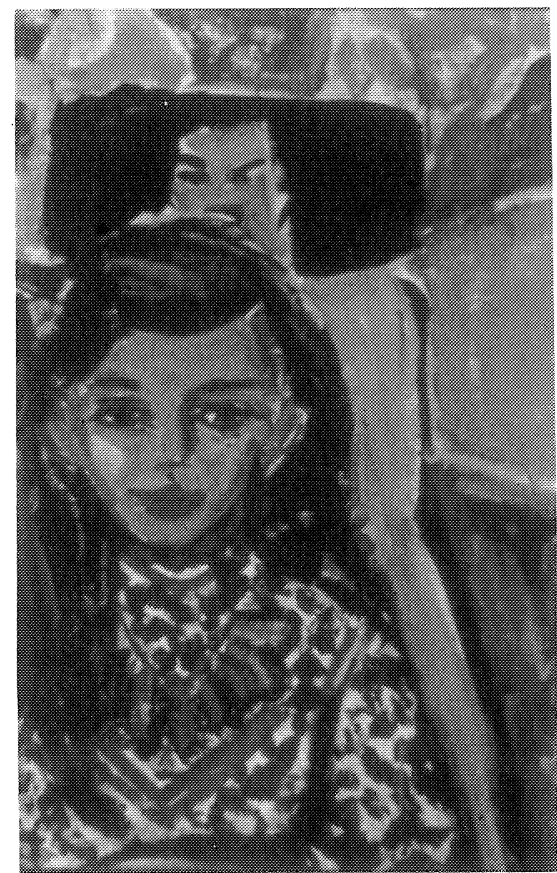

FiguRA 2. «Habitación del hotel» Edward Hopper

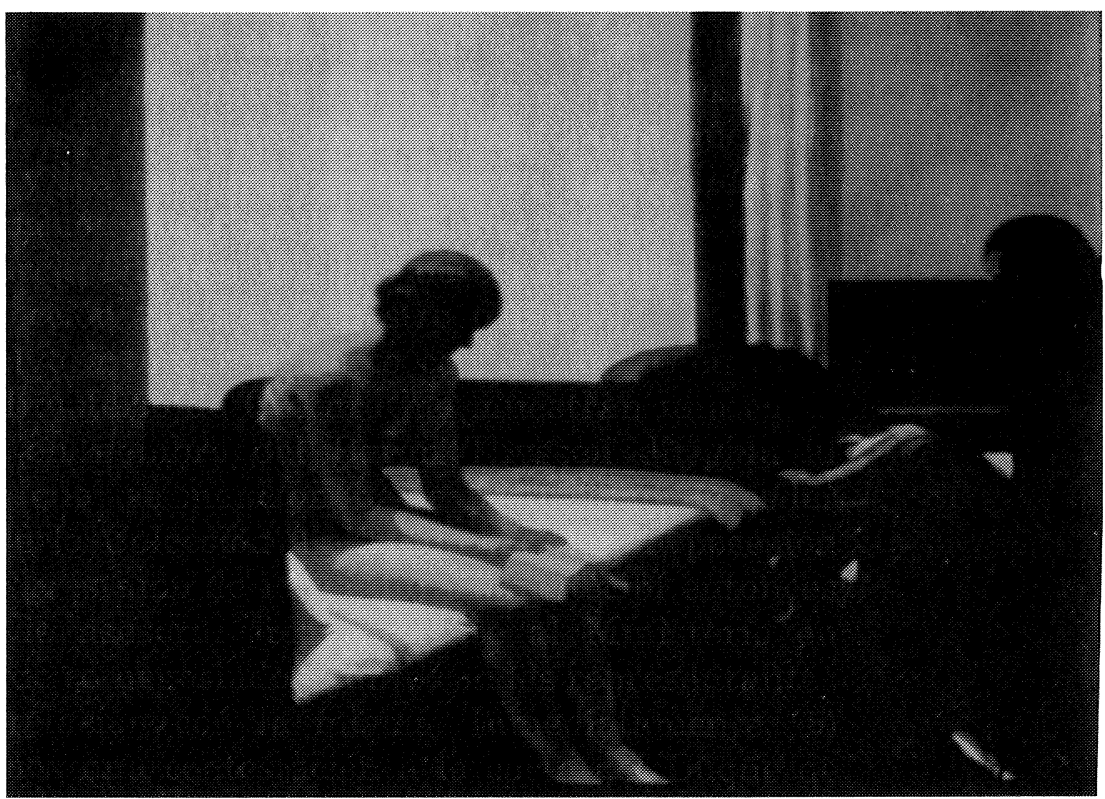




\section{Un relato: La colección Thyssen Bornemisza en España}

FIGURA 3. «Enrique VIII de Inglaterra» Hans Hobbein, el Joven

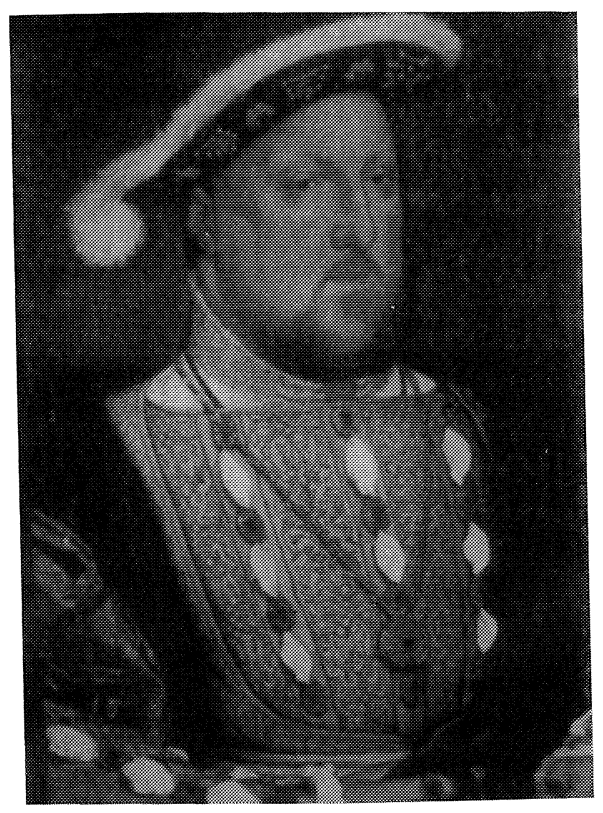

FIGURA 4. "Santa Catalina de Alejandría» Caravaggio

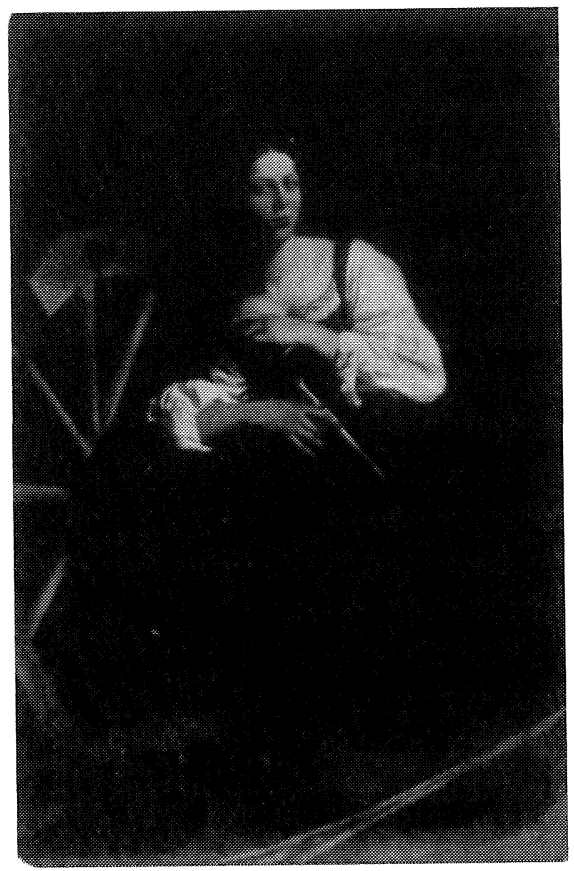

